\title{
Pulmonary veins epicardial isolation with high- intensity focused ultrasounds for the treatment of non-primary atrial fibrillation
}

\begin{abstract}
Background: Atrial fibrillation (AF) can be treated surgically by using high intensityfocused ultrasounds as an energy source with the Epicor system. The objectives of the present study are: first, to evaluate the effectiveness and safety of that technique at one year of follow-up; second, to establish the differences of the results depending on whether AF is paroxysmal or chronic and third, according to the underlying heart disease.
\end{abstract}

Methods: We treated 59 patients with the Epicor system, by doing a pulmonary veins epicardial ablation in patients with no primary AF operated on due to their cardiac disease. Patients were divided into two groups, one with 20 patients with paroxysmal AF and another with 39 chronic AF. Presence of sinus rhythm (SR) at first year was the primary endpoint and it was established according to EKG.

Results: The effectiveness to maintain SR was $67.8 \%$ in the operating room (OR), $54.4 \%$ at discharge, 54.7 at one month, $62 \%$ at six months and $65.3 \%$ at one year. No significant differences were found according to the underlying disease or the type of AF, although the results were better when AF was paroxysmal (at one year maintained SR up to $87.5 \%$ of mitral patients, $100 \%$ of aortic patients and $66.7 \%$ of ischemic patients). There were no technique-related serious complications.

Conclusion: Epicardial surgical ablation of the pulmonary veins with high-intensity focused ultrasounds is safe and shows good results, especially in patients with paroxysmal $\mathrm{AF}$, and it should be offered when a surgical procedure is scheduled as a part of the surgical treatment

Keywords: ablation, arrhythmia, atrial fibrillation, ultrasound, veins
Volume 9 Issue 4 - 2017

Bernardo Romero Ferrer,' Josep Lupón, ${ }^{2}$ Irma Casas García, ${ }^{3}$ Enrique Moret Ruiz, ${ }^{4}$ Andrea Colli, ${ }^{5}$ Luis Delgado Ramis,' Claudio Fernández Gallego,' Elisabet Berastegui García,' Ignasi Julià Amill,' Sara Badia Gamarra,' Maria-Luisa Cámara Rosell,' Francisco-Javier Ruyra Baliard, ${ }^{6}$ Benjamí Oller Sales ${ }^{6}$

'Division of Cardiac Surgery, Hospital Universitari Germans Trias i Pujol, Spain

${ }^{2}$ Division of Cardiology, Hospital Universitari Germans Trias i Pujol, Badalona, Spain, School of Medicine, Universitat Aut ${ }^{3}$ Department of Preventive Medicine, School of Medicine, Universitat Aut

${ }^{4}$ Department of Surgery, School of Medicine, Universitat Aut ${ }^{5}$ Division of Cardiology and Thoracic and Vascular Sciences, Padova University, Italy

${ }^{6}$ Department of Surgery, School of Medicine, Universitat Autònoma de Barcelona, Spain

Correspondence: Bernardo Romero-Ferrer, Servicio de Cirugía Cardíaca, Hospital Universitari Germans Trias i Pujol, Carretera de Canyet, s/n, 08916 Badalona, Spain, Tel 34654894796, Email bernatromeroferrer@hotmail.com
Abbreviations: AF, atrial fibrillation; SR, sinus rhythm; OR, operating room; HIFU, high-intensity focused ultrasounds; SD, standard deviation; LA, left atrium; Mm, millimeters; EF, ejection fraction; NSR, no sinus rhythm; SP, surgical procedure

\section{Introduction}

Atrial fibrillation (AF) is the most frequent cardiac arrhythmia. Several studies, including the Framingham one, show the association between $\mathrm{AF}$ and increased risk of mortality and morbidity. ${ }^{2-8}$ It is also known that patients who present for cardiac surgery and who already have a history of AF, have a worse prognosis if it is not treated. ${ }^{9-11} \mathrm{We}$ know that valvular or coronary surgery is not sufficient per se to cure the arrhythmia. Thus, Raine reports that of 92 patients operated on for chronic mitral insufficiency and AF, only $8.5 \%$ were in sinus rhythm (SR) after the intervention. ${ }^{12}$ Haissaguerre took a fundamental step in understanding the pathogenesis of AF by demonstrating that it was generated by focal points as a trigger around pulmonary veins. ${ }^{13}$ From the surgical point of view, Cox and his team developed a first effective surgical treatment for $\mathrm{AF}^{14,15}$ with high efficiency but it was technically difficult, added extra time to surgery and carried out morbidity. In fact, the success rate reported by the same Cox is over $90 \%$. Other authors, taking advantage of the development of new technologies and energy sources, adapted the original procedure making it simpler and less invasive.

Given the need to simplify the surgical procedure, and thanks to
Haissaguerre's work, the ablation of the pulmonary veins has raised as an alternative. The main advantage is that ablation can be done directly from the epicardium, without opening heart cavities or adding bypass or surgery time. There are different sources of energy to do the ablation, such as radiofrequency, cryoablation, microwave, laser or high intensity ultrasound (HIFU). HIFU release energy in a focal manner, avoiding the damage of surrounding tissues of the heart. The energy used is acoustic and managed in a targeted manner reaching 10 millimeters from the transducer surface. Beyond this point the energy is dissipated within the left atrial cavity without causing damage to near structures. ${ }^{16}$

The objectives of this study are four: First, to evaluate the effectiveness of exclusive epicardial ablation of pulmonary veins with HIFU in patients with non- primary AF in our environment. Second, to establish, if any, differences in the results between paroxysmal $\mathrm{AF}$ and chronic AF patients. Third, to establish, if any, differences in the results obtained according to the cardiac underlying disease of patients. And fourth, to assess the safety of the technique in terms of related complications and mortality.

\section{Material and methods}

This is a prospective observational study of a cohort of patients with non-primary AF operated on due to their underlying heart disease, in which an epicardial ablation of pulmonary veins with HIFU using the Epicor system has been added to surgical procedure. 


\section{Study population}

Ours is a tertiary center which began its program of Cardiac Surgery in 2000. From March 2006 to March 2013, 71 patients with AF have been treated. We used different techniques and, specifically, 59 patients have been treated with HIFU using the Epicor system. What has been done is to complete the surgical treatment of the underlying disease with surgical ablation of arrhythmia.

\section{Ethical considerations}

This study was approved by the Ethics Committee Research Center, code Ultrasons01 / 2014, reference CEI PI-14-032 and has been prepared in accordance with international recommendations on clinical research (Declaration of Helsinki of the World Medical Association). All patients received verbal and written information about the surgery planned and the additional ablation procedure. All patients signed an informed consent.

\section{Postoperative protocol}

Until discharge, heart rhythm was monitored and recorded by means of an EKG. In the absence of formal contraindications, amiodarone was administered to patients for a month and anticoagulation with oral anticoagulants a minimum of 6months. External electrical cardioversion was not considered necessary during admission unless patients poorly tolerated arrhythmia. Once discharged, patients were followed-up at one month, six months and then every year. The heart rate was established according to surface EKG, therefore, there are not Holter monitoring or other systems data available. All visits were made in the Outpatient Clinic of Cardiac Surgery Division. The primary endpoint is the maintenance of SR to the first year of follow- up. In addition, data are available for maintenance of SR at 2 and 3years of intervention in certain patients.

\section{Statistical analysis}

Data were treated with SPSS version 19 software. Continuous variables followed a normal distribution and are described as mean and standard deviation. Categorical variables were described as proportions. During follow-up, heart rate was divided into two groups for analysis, the first consisting of patients with SR and the second by patients with no SR, whether or not AF. Differences between groups were stablished with the Student's $t$ test for continuous variables $x^{2}$ test or Fisher's exact test, when indicated, for categorical variables. A result of less than 0.05 was considered statistically significant. The relationship between the variables and maintenance of SR at first year was stablished by univariate and multivariate logistic regression analysis. A Kaplan-Meier curve (AF drop in follow-up) for the overall group of patients was built. The log-rank was used as a comparison test.

\section{Results and discussion}

\section{Preoperative data}

We treated a total of 59 patients, who were divided into two groups to assess differences in the results. The first group consists of 20 patients with a history of paroxysmal AF (33.9\%). The second group consists of the 39 patients $(66.1 \%)$ with chronic AF (persistent 5 [8.5\%] and 34 [57.6\%] permanent). ${ }^{17}$ The clinical characteristics of patients are shown in Table 1. Significant differences in the surgical risk and taking calcium channel blockers and antiplatelet drugs were found.

Table I Differences between the group of patients with paroxysmal AF and the group of patients with chronic AF Results are expressed as mean and standard deviation or as number and percentage

\begin{tabular}{|c|c|c|c|}
\hline & Paroxysmal AF $(n=20)$ & Chronic AF $(n=39)$ & $\mathbf{p}$ \\
\hline Age (years) & 66 (SD 9,09) & $65,28(\mathrm{SD} \mid 2,10)$ & $0,8 \mid 8 I$ \\
\hline Gender (men) & $15(75 \%)$ & $25(64,10 \%)$ & 0,3962 \\
\hline \multicolumn{4}{|l|}{ Pathology } \\
\hline Mitral & $9(45 \%)$ & $19(48,70 \%)$ & 0,7872 \\
\hline Aortic & $5(25 \%)$ & $12(30,80 \%)$ & 0,6432 \\
\hline Coronary & $6(30 \%)$ & $5(12,80 \%)$ & 0,1592 \\
\hline Congenital & 0 & $3(7,70 \%)$ & 0,5442 \\
\hline AF length (months) & 88,80 (SD I53,47) & 44,39 (SD 62,39) & 0,2531 \\
\hline LA size $(\mathrm{mm})$ & $48,56(S D 6,32)$ & $51,76(S D 8,13)$ & 0,1521 \\
\hline $\mathrm{EF} \%$ & 58,60 (SD I2,07) & 55,33 (SD I4,59) & 0,3931 \\
\hline Euroscore & 4,95 (SD I,60) & 6,05 (SD 2,5I) & $0,046 I$ \\
\hline Logistic Euroscore & 4,07 (SD I,92) & 6,88 (SD 5,39) & 0,0051 \\
\hline \multicolumn{4}{|l|}{ Treatment } \\
\hline Digoxin & 7 (35\%) & $17(43,60 \%)$ & 0,5252 \\
\hline$\beta$-blockers & $5(25 \%)$ & $12(30,80 \%)$ & 0,6432 \\
\hline Calcium channel blockers & $2(10 \%)$ & $15(38 \%)$ & 0,0222 \\
\hline Antiplatelet & $8(40 \%)$ & $3(7,7 \%)$ & 0,0032 \\
\hline Oral anticoagulants & 15 (75\%) & $33(84,60 \%)$ & 0,4832 \\
\hline Amiodarone & $7(35 \%)$ & $5(12,80 \%)$ & 0,0512 \\
\hline Percutaneous ablation & 0 & I $(2,60 \%)$ & 0,9002 \\
\hline Thromboembolism & $2(10 \%)$ & $7(17,90 \%)$ & 0,7042 \\
\hline
\end{tabular}

AF:Atrial Fibrillation; SD: Standard Deviation; LA: Left Atrium; EF: Ejection Fraction; I: Student's T test; $2: \chi 2$ test

\section{Perioperative data}

The average duration of the ablation procedure was 574 (SD 21) seconds (between 537 and 650seconds), about 9 and a half minutes, with no differences between the two groups. The average size of UltraCinch was 10.78 (SD 1,451) (8 to 13) with a mode of 11 in 16 cases $(27.1 \%)$. In patients with chronic $\mathrm{AF}$, size was significantly higher (10.20 SD1.19 vs 11.08 SD1.49, $\mathrm{p}=0.027)$. 


\section{Postoperative data}

\section{Complications and mortality}

No patient died or suffered a complication related to the ablation procedure. Supplementary Table 1 describes the complications suffered by patients during admission. During follow-up, a pacemaker was implanted in two patients (3.63\%) and five patients died $(9.09 \%)$, one due to a peripheral gangrene of the lower limbs, one due to an acute myocardial infarction, one kidney failure, and one septic shock and in the fifth patient the cause of death was not identified. Three patients had a chronic AF and two paroxysmal AF $(p=0.900)$.

Supplementary Table I Complications during admission

\begin{tabular}{lll} 
Complication & $\mathbf{n}$ & $\%$ \\
\hline Respiratory failure & 2 & $3,39 \%$ \\
Renal failure & 6 & $10,17 \%$ \\
Pericardial effusion & $\mathrm{I}$ & $\mathrm{I}, 69 \%$ \\
Cardiac tamponade & $\mathrm{I}$ & $\mathrm{I}, 69 \%$ \\
Low cardiac output & $\mathrm{I}$ & $\mathrm{I}, 69 \%$ \\
Pleural effusion & $\mathrm{I}$ & $\mathrm{I}, 69 \%$ \\
Bleeding and reoperation & $\mathrm{I}$ & $\mathrm{I}, 69 \%$ \\
Mesenteric ischemia & $\mathrm{I}$ & $\mathrm{I}, 69 \%$ \\
Adynamic ileus & $\mathrm{I}$ & $\mathrm{I}, 69 \%$ \\
Wound infection & $\mathrm{I}$ & $\mathrm{I}, 69 \%$ \\
Pacemaker & 2 & $3,39 \%$ \\
\hline
\end{tabular}

\section{Heart rhythm during follow-up}

Table 2 shows the results of the intervention at first year. Most patients $(65.3 \%)$ are in SR, especially when AF is paroxysmal, although the difference did not reach statistical significance $(\mathrm{p}=$ 0.068 ). Moreover, more than half of patients with chronic AF are also in SR. Table 3 shows the results in the other moments of follow-up, from the operating room to 3years. At all times of follow-up, there are more patients in SR than in AF. Only at first month, the difference in keeping SR between patients with paroxysmal AF and chronic AF is statistically significant. Specifically at 3years we have data on 44 patients, being $56.8 \%$ of them in SR.

Table 2 Results at first year of intervention by type of AF and underlying pathology

\begin{tabular}{|c|c|c|c|}
\hline & Sinus Rhythm & Non Sinus Rhythm & $\mathbf{p}$ \\
\hline Type of AF & & & 0,06 \\
\hline Paroxysmal & $14(82,4 \%)$ & $3(17,6 \%)$ & \\
\hline Chronic & $18(56,3 \%)$ & $14(43,7 \%)$ & \\
\hline Type of Patholog) & & & 0,72 \\
\hline Mitral & $17(70,8 \%)$ & $7(29,2 \%)$ & \\
\hline Aortic & $8(66,7 \%)$ & $4(33,3 \%)$ & \\
\hline Coronary & $5(50 \%)$ & $5(50 \%)$ & \\
\hline Congenital & $2(66,7 \%)$ & I (33,3\%) & \\
\hline
\end{tabular}

AF:Atrial Fibrillation

Table 3 Heart rhythm in operating room, at discharge, I month, 6 months, 2 years and 3 years

\begin{tabular}{|c|c|c|c|c|c|c|c|c|c|c|c|c|c|c|c|c|c|c|}
\hline \multicolumn{4}{|c|}{ Operating Room } & \multicolumn{3}{|c|}{ Discharge } & \multicolumn{3}{|c|}{ I Month } & \multicolumn{3}{|c|}{6 Months } & \multicolumn{3}{|c|}{2 Years } & \multicolumn{3}{|c|}{3 Years } \\
\hline & SR & NSR & $\mathbf{p}$ & SR & NSR & $\mathbf{p}$ & SR & NSR & $\mathbf{p}$ & SR & NSR & $\mathbf{p}$ & SR & NSR & $\mathbf{p}$ & SR & NSR & $\mathbf{P}$ \\
\hline Type of AF & & & 0,126 & & & 0,071 & & & 0,006 & & & 0,113 & & & 0,161 & & & 0.267 \\
\hline Paroxysmal & $80 \%$ & $20 \%$ & & $68,4 \%$ & $33,6 \%$ & & $84,2 \%$ & $15,8 \%$ & & $76,5 \%$ & $23,5 \%$ & & $75 \%$ & $25 \%$ & & $66,7 \%$ & $33,3 \%$ & \\
\hline Chronic & $61,5 \%$ & $38,6 \%$ & & $47,4 \%$ & $52,6 \%$ & & $41,7 \%$ & $58,3 \%$ & & $54,5 \%$ & $45,5 \%$ & & $55,2 \%$ & $44,8 \%$ & & $51,7 \%$ & $48,3 \%$ & \\
\hline $\begin{array}{l}\text { Type of } \\
\text { Pathology }\end{array}$ & & & 0.085 & & & 0,887 & & & 0,669 & & & 0,726 & & & 0,673 & & & 0,737 \\
\hline Mitral & $71 \%$ & $29 \%$ & & $57 \%$ & $43 \%$ & & $50 \%$ & $50 \%$ & & $66 \%$ & $34 \%$ & & $68 \%$ & $32 \%$ & & $64 \%$ & $36 \%$ & \\
\hline Aortic & $53 \%$ & $47 \%$ & & $53 \%$ & $47 \%$ & & $64 \%$ & $36 \%$ & & $58 \%$ & $42 \%$ & & $63 \%$ & $37 \%$ & & $45 \%$ & $55 \%$ & \\
\hline Coronary & $91 \%$ & $9 \%$ & & $55 \%$ & $45 \%$ & & $55 \%$ & $45 \%$ & & $63 \%$ & $37 \%$ & & $44 \%$ & $56 \%$ & & $50 \%$ & $50 \%$ & \\
\hline Congenital & $33,3 \%$ & $66,7 \%$ & & $33,3 \%$ & $66,7 \%$ & & $33.30 \%$ & $66,7 \%$ & & $33,3 \%$ & $66,7 \%$ & & $66,7 \%$ & $33, \%$ & & $66,7 \%$ & $33,3 \%$ & \\
\hline
\end{tabular}

AF:Atrial Fibrillation; SR: Sinus Rhythm; NSR: Non Sinus Rhythm

Supplementary Table 2 Patients in sinus rhythm at first year according to the underlying disease and type of AF

\begin{tabular}{lllll}
\hline Pathology & Paroxysmal AF & Chronic AF & Overall & P \\
\hline \multirow{2}{*}{ Mitral } & 7 & 10 & 17 & 0,352 \\
& $(87,5 \%)$ & $(62,5 \%)$ & $(70,8 \%)$ & \\
Aortic & 3 & 5 & 8 & $0,49 \mid$ \\
& $(100 \%)$ & $(55,5 \%)$ & $(66,6 \%)$ & \\
Coronary & 4 & 1 & 5 & 0,524
\end{tabular}

Table 4 Relationship of variables with the maintenance of sinus rhythm at first year. Univariate analysis

\begin{tabular}{llll}
\hline & OR & $\mathbf{9 5 \%} \mathbf{C l}$ & $\mathbf{P}$ \\
\hline $\begin{array}{l}\text { Paroxysmal AF } \\
\text { Pathology }\end{array}$ & 3,63 & $0,87-15,15$ & 0,068 \\
Mitral & & & 0,721 \\
Aortic & 1 & & \\
Coronary & 0,82 & $0,18-3,64$ & 0,798 \\
Congenital & 0,41 & $0,09-1,88$ & 0,253 \\
Age & 0,82 & $0,06-10,61$ & 0,882 \\
Male gender & 0,93 & $0,87-1,00$ & 0,052 \\
Euroscore & 3,41 & $0,65-17,8$ & 0,146 \\
AF length (months) & 0,69 & $0,51-0,93$ & 0,016 \\
LA size (mm) & 0,94 & $0,99-1,00$ & 0,759 \\
EF & 0,86 & $0,75-0,97$ & 0,017 \\
\hline
\end{tabular}

Citation: Ferrer BR, Lupón J, García IC, et al. Pulmonary veins epicardial isolation with high-intensity focused ultrasounds for the treatment of non-primary atrial fibrillation.J Cardiol Curr Res. 2017;9(4): I-6. DOI: I0.15406/jccr.2017.09.00329 
Table Continued..

\begin{tabular}{llll} 
& OR & $\mathbf{9 5 \%} \mathbf{C l}$ & $\mathbf{P}$ \\
\hline CPB time (minutes) & 0,99 & $0,97-1,00$ & 0,279 \\
Cross clamp time (minutes) & 0,98 & $0,96-1,01$ & 0,321 \\
Length or surgical procedure (minutes) & 0,99 & $0.96-1,00$ & 0,384 \\
\hline
\end{tabular}

OR: Odds Ratio; Cl: Confidence Interval;AF:Atrial Fibrillation; LA: Left Atrium; EF: Ejection Fraction; CPB: Cardiopulmary Bypass

Table 5 Relationship of variables with the maintenance of sinus rhythm at first year. Multivariate logistic regression analysis

\begin{tabular}{llll} 
& OR & $\mathbf{9 5 \%} \mathbf{C l}$ & $\mathbf{p}$ \\
\hline Paroxysmal AF & 5,46 & $\mathrm{I}, \mathrm{I} 2-26,5 \mathrm{I}$ & 0,035 \\
Age & $0,9 \mathrm{I}$ & $0,85-0,99$ & 0,027 \\
Male gender & 7,36 & $\mathrm{I}, 10-49,22$ & 0,039 \\
LA size $(\mathrm{mm})$ & 0,88 & $0,76-\mathrm{I}, 0 \mathrm{I}$ & 0,084 \\
EF & $\mathrm{I}, 03$ & $0,97-\mathrm{I}, 09$ & 0,315 \\
Euroscore & $0,9 \mathrm{I}$ & $0,48-\mathrm{I}, 70$ & 0,760
\end{tabular}

AF: Atrial Fibrillation; LA: Left Atrium; EF: Ejection Fraction; OR: Odds Ratio; $\mathrm{Cl}$ : Confidence Interval

There were no differences in the primary outcome at first year according to the underlying heart disease (Table 2) or other followup time (Table 3). Within each pathology there was no difference between patients with paroxysmal AF and chronic AF, not only at first year. It's noteworthy that all aortic patients with paroxysmal AF remain in SR at first year, a result that is also kept the second year. But it is also noteworthy that $87.5 \%$ of mitral patients with paroxysmal AF are in SR (Supplementary Table 2). Especially discreet are the results of coronary patients, even with paroxysmal AF.

\section{Univariate and multivariate analysis and Kaplan-Meier curve}

In univariate logistic regression analysis, only Euroscore and size of the left atrium were significantly related to the maintenance of SR at first year, while age bordered statistical significance (Table 4). In multivariate analysis, the variables that were significant were the type of AF, age and gender (Table 5). Figure 1 shows the Kaplan-Meier curve (AF relapse during follow-up) for the overall group of patients.

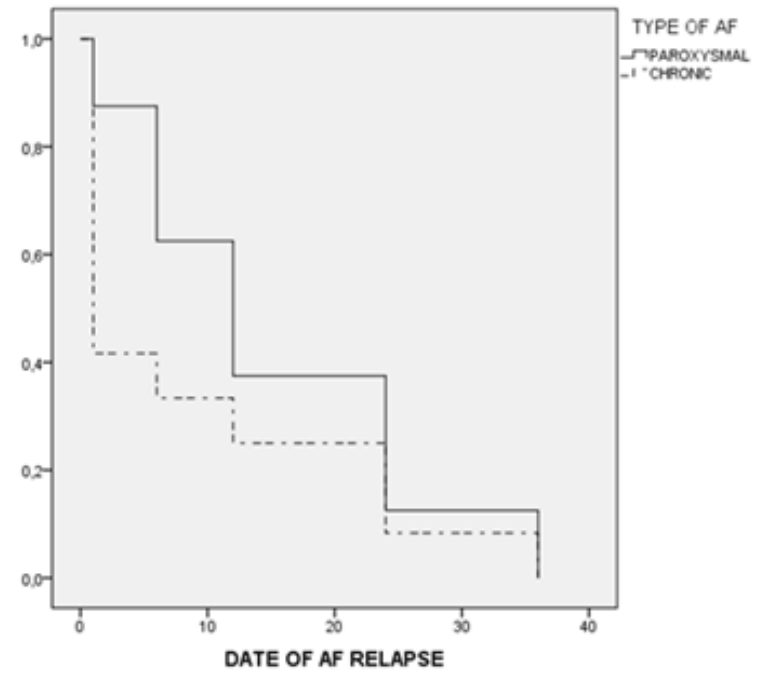

Figure I Kaplan-Meier curve (AF relapse during follow-up).

Log Rank 0,48I Among patients with SR after the ablation procedure, we can see that the recurrence of AF during follow-up is earlier in those patients with previous chronic AF compared to patients with previous paroxysmal AF, although the differences are not statistically significant.

\section{Discussion}

$\mathrm{AF}$ is a common condition among patients who have to undergo cardiac surgery and it is associated with increased surgical morbidity and mortality. ${ }^{18-21}$ In our study should be noted that the ablation procedure is added to the surgical procedure that each patient needs for his cardiac illness. Also, it does not increase the time of surgery, since ablation is performed while the surgeon prepares the patient for cardiopulmonary bypass. Overall, in all follow-up times there are more patients who maintain SR, specifically at first year $65.3 \%$ of patients are in SR. Our results are in line with those of the Spanish National Registry and at three years they are even better. ${ }^{22}$ And they are consistent with data from other studies. Thus, Schopka reports a maintenance SR at one year of $62 \% .{ }^{23}$ Camm published a review of the most important articles in the literature with maintenance of SR after surgery and ablation procedure ranging from 60 and $82 \% .{ }^{24}$ Even so, our results are lower than those shown in the best series published also using HIFU as an energy source..$^{25,26}$ Groh reports maintenance of SR of $84.4 \%$ of patients at first year. Ninet's study has a follow-up of six months, reaching maintenance of SR in $85 \%$ of patients and $100 \%$ when $\mathrm{AF}$ is paroxysmal.

We have also to keep in mind that our strategy has been to make only an ablation procedure around the pulmonary veins, regardless of the underlying disease and the need or not to open heart chambers. Therefore, it is the simplest ablation procedure that can currently be offered, without adding new lines of ablation, which could explain the better results of other studies. In this sense the FAST study is particularly interesting. ${ }^{27,28}$ Although radiofrequency energy source and not the HIFU was used in this study, no difference between surgical patients receiving mitral line mitral ablation compared to those who only received an ablation of the pulmonary veins was found, which would support our ablation strategy. Gillinov also studies the results of surgical radiofrequency ablation or cryoablation in mitral patients with paroxysmal AF. He concludes that the isolation of the pulmonary veins may be sufficient in these patients, especially when the AF is recent. ${ }^{29}$

In our series, the scenario is more favorable in the case of patients with paroxysmal AF. Thus, at first postoperative month and first year, $82.4 \%$ of patients with paroxysmal AF maintain SR. For patients with chronic AF, they maintain SR at first month up to $41.7 \%$, at 6 months $54.5 \%$ and at first year $56.3 \%$. The literature also shows that the results are better in cases of paroxysmal AF. In Camm study up to $47 \%$ of patients with chronic AF maintain SR, but up to $85 \%$ of patients with paroxysmal AF remain in. ${ }^{15,24-30}$ Pizón's and Davies' studies are the latest published using HIFU. Davies reports, at two years, $49 \%$ of patients maintaining SR, but according to the type of AF, patients with paroxysmal AF kept SR up to $81 \%$ of cases, patients with persistent AF up to $56 \%$ and patients with permanent AF up to $18 \%$ of cases. ${ }^{31}$ Pizón studied 78 patients. At 3 and 6 months remained in SR 66.7\% and $100 \%$ of patients with paroxysmal AF, $100 \%$ and $90 \%$ of patients with persistent $\mathrm{AF}$ and $33.3 \%$ and $56 \%$ of patients with permanent AF. As predictors of the ablation outcome, Pizón found that patients who maintained sinus SR had a smaller left atrial diameter and a smaller left atrial area. ${ }^{32}$ In our study, the variables that were related to maintenance of SR at first year were Euroscore and the size of left 
atrium in the univariate analysis, while in multivariate analysis were paraxismal $\mathrm{AF}$, age and male gender.

If we look at the results according to the underlying conditions of patients, in all cases but coronary patients at the end of surgery, $\mathrm{SR}$ is always more incident when AF is paroxysmal. In the case of coronary patients, our results are lower than those of the best series published, like Groh, reporting $85 \%$ of patients with coronary disease who maintain SR at one year of follow-up. ${ }^{33}$ Even so, more than half of coronary patients with paroxysmal AF RS remain in SR at first year in our study. We are especially interested in the results obtained in patients with mitral and aortic pathology. In fact, $100 \%$ of aortic patients with paroxysmal AF remain in SR until the second year and at third year up to $66.7 \%$ are in SR. And for mitral patients with paroxysmal AF, up to $90 \%$ are in SR at first year and $75 \%$ at second and third year.

In our series, no patient died or suffered any complication related to the ablation procedure. The most common complication was renal failure and no cases of stroke or thromboembolism were observed. In a recent meta-analysis, Phan concludes that adding an ablation procedure to mitral surgery significantly increases the rate of SR, without increasing mortality rates, need for permanent pacemaker, stroke or thromboembolism. ${ }^{34}$ Thus, the observed complications are those expected after a similar cardiac intervention but without the procedure ablation. ${ }^{35}$ The results of this study are in line with other studies which show that adding the AF ablation procedure to the planned surgery does not increase the surgical risk. ${ }^{2,36}$ Thereby, after the study of complications and mortality we can say that the technique is safe.

Although the difference is statistically significant only at first month, at one the year is quite significant. And although it is not significant neither at second nor at third year, this surgical ablation technique cannot be denied a priori to a patient presenting with a chronic AF, since at least half of treated patients will remain in SR.

\section{Looking to the future}

We can offer hybrid procedures to our patients, offering cardiologists and surgeons the opportunity of working together, combining in one procedure, a surgical epicardial ablation and a minimally invasive percutaneous endocardial ablation, using the best of both techniques. Surgeons would create ablation lines and cardiologists would evaluate and complete them when necessary, for example, when a line was not transmural. HIFU would be an excellent bet in this regard. This concept of hybrid procedure was proposed by $\mathrm{Pak}^{37}$ and other authors have already described their results. ${ }^{38-41}$

\section{Limitations}

The small number of patients included difficults to find significant differences. Also, this is an observational study, not a randomized experimental one because it does not compare to a control group. But the most important limitation is that the heart rate during followup has only been based on the surface EKG. Therefore, no data are available derived from Holter monitoring or implantable devices. It is possible that the incidence of patients in SR may be overestimated.

\section{Conclusion}

Epicardial surgical ablation of the pulmonary veins with HIFU is safe and shows good results at first year, especially in patients with paroxysmal AF and should be offered to patients with surgical indication for their underlying condition.

\section{Acknowledgements}

Authors would like to thank the Divisions of Cardiac Surgery and Cardiology at Hospital Universitari Germans Trias i Pujol for their support.

\section{Conflicts of interest}

Author declares there are no conflicts of interest.

\section{Funding}

None.

\section{References}

1. Lloyd-Jones D, Adams RJ, Brown TM, et al. Heart disease and stroke statistics--2010 update: a report from the American Heart Association. Circulation. 2010;121(7):e46-e215.

2. Ad N, Henry L, Hunt S, et al. Do we increase the operative risk by adding the Cox Maze III procedure to aortic valve replacement and coronary artery bypass surgery? J Thorac Cardiovasc Surg. 2012;143(4):936-944.

3. Jabre $\mathrm{P}$, Jouven $\mathrm{X}$, Adnet $\mathrm{F}$, et al. Atrial fibrillation and death after myocardial infarction: a community study. Circulation. 2011;123(19):2094-2100.

4. Lau DH, Alasady M, Brooks AG, et al. New-onset atrial fibrillation and acute coronary syndrome. Expert Rev Cardiovasc Ther 2010;8(7):941-948.

5. Kannel WB, Abbott RD, Savage DD, et al. Coronary heart disease and atrial fibrillation: the Framingham Study. Am Heart $J$. 1983;106(2):389-396

6. Lubitz SA, Magnani JW, Ellinor PT, et al. Atrial fibrillation and death after myocardial infarction: risk marker or causal mediator?. Circulation. 2011;123(19):2063-2065.

7. Krahn AD, Manfreda J, Tate RB, et al. The natural history of atrial fibrillation: incidence, risk factors, and prognosis in the Manitoba Follow-Up Study. Am J Med. 1995;98(5):476-484.

8. Psaty BM, Manolio TA, Kuller LH, et al. Incidence of and risk factors for atrial fibrillation in older adults. Circulation. 1997;96(7):2455-2461.

9. Ngaage DL, Schaff HV, Barnes SA, et al. Prognostic implications of preoperative atrial fibrillation in patients undergoing aortic valve replacement: is there an argument for concomitant arrhythmia surgery? Ann Thorac Surg. 2006;82(4):1392-1399.

10. Kalavrouziotis D, Buth KJ, Vyas T, et al. Preoperative atrial fibrillation decreases event-free survival following cardiac surgery. Eur $J$ Cardiothorac Surg. 2009;36(2):293-299.

11. Quader MA, McCarthy PM, Gillinov AM, et al. Does preoperative atrial fibrillation reduce survival after coronary artery bypass grafting?. Ann Thorac Surg. 2004;77(5):1514-1522.

12. Raine D, Dark J, Bourke JP. Effect of mitral valve repair/ replacement surgery on atrial arrhythmia behavior. $J$ Heart Valve Dis. 2004;13(4):615-621.

13. Haïssaguerre M, Marcus FI, Fischer B, et al. Radiofrequency catheter ablation in unusual mechanisms of atrial fibrillation: report of three cases. J Cardiovasc Electrophysiol. 1994;5(9):743-751.

14. Cox JL, Canavan TE, Schuessler RB, et al. The surgical treatment of atrial fibrillation. II. Intraoperative electrophysiologic mapping and description of the electrophysiologic basis of atrial flutter and atrial fibrillation. J Thorac Cardiovasc Surg. 1991;101(3):406-426.

15. Cox JL, Schuessler RB, Boineau JP. The surgical treatment of atrial fibrillation. I. Summary of the current concepts of the mechanisms of atrial flutter and atrial fibrillation. $J$ Thorac Cardiovasc Surg. 1921;101(3):402-405. 
16. Lee LA, Simon C, Bove EL, et al. High intensity focused ultrasound effect on cardiac tissues: potential for clinical application. Echocardiography. 2000;17(6 Pt 1):563-566.

17. Camm AJ, Kirchhof P, Lip GY, et al. Guidelines for the management of atrial fibrillation: the Task Force for the Management of Atrial Fibrillation of the European Society of Cardiology (ESC). Eur Heart J. 2010;31(19):2369-2429.

18. Eguchi K, Ohtaki E, Matsumura T, et al. Pre-operative atrial fibrillation as the key determinant of outcome of mitral valve repair for degenerative mitral regurgitation. Eur Heart J. 2005;26(18):1866-1872.

19. Saxena A, Dinh D, Dimitriou J, et al. Preoperative atrial fibrillation is an independent risk factor for mid-term mortality after concomitant aortic valve replacement and coronary artery bypass graft surgery. Interact Cardiovasc Thorac Surg. 2013;16(4):488-494.

20. Saxena A, Dinh DT, Reid CM, et al. Does preoperative atrial fibrillation portend a poorer prognosis in patients undergoing isolated aortic valve replacement? A multicentre Australian study. Can J Cardiol. 2013;29(6):697-703.

21. Khargi K, Hutten BA, Lemke B, et al. Surgical treatment of atrial fibrillation; a systematic review. Eur J Cardiothorac Surg . $2005 ; 27(2): 258-265$.

22. Reyes G, Ruyra X, Valderrama F, et al. High intensity focused ultrasound ablation for atrial fibrillation: results from the National Spanish Registry. Minerva Cardioangiol. 2015;64(5):501-506.

23. Schopka S, Schmid C, Keyser A, et al. Ablation of atrial fibrillation with the Epicor system: a prospective observational trial to evaluate safety and efficacy and predictors of success. J Cardiothorac. 2010;5:34.

24. Camm CF, Nagendran M, Xiu PY, et al. How effective is cryoablation for atrial fibrillation during concomitant cardiac surgery? Interact Cardiovasc Thorac Surg. 2011; 13(4):410-414.

25. Groh MA, Binns OA, Burton HG 3rd, et al. Ultrasonic cardiac ablation for atrial fibrillation during concomitant cardiac surgery: long-term clinical outcomes. Ann Thorac Surg. 2007;84(6):1978-1983.

26. Ninet J, Roques X, Seitelberger R, et al. Surgical ablation of atrial fibrillation with off-pump, epicardial, high-intensity focused ultrasound: results of a multicenter trial. J Thorac Cardiovasc Surg. 2005;130(3):803-809.

27. Castellá M. Surgical ablation in atrial fibrillation: in which patients? Heart. 2013;99(12):888-892.

28. Boersma LV, Castella M, van Boven W, et al. Atrial fibrillation catheter ablation versus surgical ablation treatment (FAST): a 2-center randomized clinical trial. Circulation . 2005;25(1):23-30.

29. Gillinov AM, Bakaeen F, McCarthy PM, et al. Surgery for paroxysmal atrial fibrillation in the setting of mitral valve disease: a role for pulmonary vein isolation?. Ann Thorac Surg. 2006;81(1):19-26.
30. Gillinov AM, Bhavani S, Blackstone EH, et al. Surgery for permanent atrial fibrillation: impact of patient factors and lesion set. Ann Thorac Surg. 2006;82(2):502-513.

31. Davies EJ, Bazerbashi S, Asopa S, et al. Long-term outcomes following high intensity focused ultrasound ablation for atrial fibrillation. $J$ Card Surg. 2014;29(1):101-107.

32. Pizon M, Friedel N, Pizon M, et al. Impact of epicardial ablation of concomitant atrial fibrillation on atrial natriuretic peptide levels and atrial function in 6 months follow-up: does preoperative ANP level predict outcome of ablation?. J Cardiothorac Surg. 2013;8:218.

33. Groh MA, Binns OA, Burton $\mathrm{HG}$ 3rd, et al. Epicardial ultrasonic ablation of atrial fibrillation during concomitant cardiac surgery is a valid option in patients with ischemic heart disease. Circulation. 2008;118(14 Suppl):S78-S82.

34. Phan K, Xie A, Tian DH, et al. Systematic review and meta-analysis of surgical ablation for atrial fibrillation during mitral valve surgery. Ann Cardiothorac Surg . 2014;3(1):3-14.

35. Wisser W, Seebacher G, Fleck T, et al. Permanent chronic atrial fibrillation: is pulmonary vein isolation alone enough? Ann Thorac Surg. 2007; 84(4):1151-1157.

36. Fukunaga S, Hori $\mathrm{H}$, Ueda $\mathrm{T}$, et al. Effect of surgery for atrial fibrillation associated with mitral valve disease. Ann Thorac Surg. 2008;86(4):1212-1217.

37. Pak HN, Hwang C, Lim HE, et al. Hybrid epicardial and endocardial ablation of persistent or permanent atrial fibrillation: a new approach for difficult cases. J Cardiovasc Electrophysiol. 2007;18(9):917-923.

38. Krul SP, Driessen AH, van Boven WJ, et al. Thoracoscopic videoassisted pulmonary vein antrum isolation, ganglionated plexus ablation, and periprocedural confirmation of ablation lesions: first results of a hybrid surgical-electrophysiological approach for atrial fibrillation. Circ Arrhythm Electrophysiol. 2011;4(3):262-270.

39. Mahapatra S, LaPar DJ, Kamath S, et al. Initial experience of sequential surgical epicardial-catheter endocardial ablation for persistent and longstanding persistent atrial fibrillation with long-term follow-up. Ann Thorac Surg. 2011;91(6):1890-1898.

40. Pison L, La MM, van OJ, et al. Hybrid thoracoscopic surgical and transvenous catheter ablation of atrial fibrillation. J Am Coll Cardiol. 2012;60(1):54-61.

41. Bisleri G, Rosati F, Bontempi L, et al. Hybrid approach for the treatment of long-standing persistent atrial fibrillation: electrophysiological findings and clinical results. Eur J Cardiothorac Surg. 2013;44(5):919-923. 